\title{
The Modified Electromagnetism and the Emergent Longitudinal Wave
}

\author{
A. I. Arbab ${ }^{1} \&$ M. Al-Ajmi \\ ${ }^{1}$ Department of Physics, Faculty of Science, University of Khartoum, P.O. Box 321, Khartoum 11115, Sudan \\ ${ }^{2}$ Department of Physics, College of Science, Sultan Qaboos University, P.O. Box 36, Alkhod 123, Oman \\ Correspondence: M. Al-Ajmi, Department of Physics, College of Science, Sultan Qaboos University , P.O. Box 36, \\ Alkhod 123, Oman. E-mail: mudhahir@squ.edu.om
}

Received: March 5, 2018

Accepted: March 14, 2018

Online Published: March 30, 2018

doi:10.5539/apr.v10n2p45

URL: https://doi.org/10.5539/apr.v10n2p45

\begin{abstract}
The classical theory of electromagnetism has been revisited and the possibility of longitudinal photon wave is explored. It is shown that the emergence of longitudinal wave is a consequence of Lorenz gauge (condition) violation. Proca, Vlaenderen \& Waser and Arbab theories are investigated. The different approaches are compared to each other and the relevant equations are combined. The telegrapher's equation can be obtained and with a specific choice of the function a Klein-Gordon equation of massive scalar field can be obtained. When the Lorentz gauge is violated by introducing the first order time derivative the emergence of the photon mass and the relevant longitudinal wave for the electromagnetic wave is apparnt.
\end{abstract}

Keywords: Einstein, Brans-Dicke, Newton, Gravitation, Mach, Sciama

\section{Introduction}

Maxwell's equations are one of the biggest edifices that mankind had come to establish. Maxwell unified electromagnetism with light in a set of consistent equation dubbed in Maxwell equation. These equation prematurely predicted that speed of light in vacuum is constant. In an elegant theory, Salam, Weinberg and Glashow unified electromagnetism with weak interaction (Glashow, 1961; Weinberg, 1967; Salam, 1962). The ultimate goal of unifying all interactions into a single universal theory is underway. Some theoretical prejudices advocated by Proca in extending Maxwell's theory to encompass massive photons was expounded in 1967 (Proca, 1936). This later theory will be of importance, at cosmic scale, as some compelling investigation usher in that direction. Of these investigation is the missing mass (dark matter) in the universe. It is remarked by Schrodinger that if the photon had a mass then the Black-Body formula will be modified and the energy density of these photons would be $3 / 2$ times the ordinary one (Bass \& Schr?dinger, 1955). This is because these photon would have a longitudinal polarization besides the transverse one. Longitudinal waves have been shown to have high penetration power. This character rendered them to be of great importance and will find wider applications in communication systems. Some endeavors to generalize Maxwell's equations to remedy these shortcoming have been proposed by following a quaternionic generalizations. In the framework of these attempts, the shortcoming present in the original Maxwell's formulation can be avoided (Arbab \& Satti, 2009). In this work, we will outline the different approaches and explore these interrelations. We will see that whenever the Lorenz gauge (condition) is violated, longitudinal waves will be inevitable. We also analyze the status of the experimental evidence supporting the presence of these waves.

\section{Maxwell's Equation}

Maxwell's equations describe the behavior of the electric and magnetic fields. These equations are (Griffiths, 1987)

$$
\vec{\nabla} \cdot \vec{E}=\frac{\rho}{\varepsilon_{0}}, \quad \vec{\nabla} \cdot \vec{B}=0,
$$

and

$$
\vec{\nabla} \times \vec{E}=-\frac{\partial \vec{B}}{\partial t}, \quad \vec{\nabla} \times \vec{B}=\mu_{0} \vec{J}+\frac{1}{c^{2}} \frac{\partial \vec{E}}{\partial t}
$$

\section{Vector and Scalar Potential}

The electric and magnetic fields are defined as

$$
\vec{E}=-\vec{\nabla} \varphi-\frac{\partial \vec{A}}{\partial t}, \quad \vec{B}=\vec{\nabla} \times \vec{A}
$$


It is believed that vector and scalar potentials, $\vec{A}$ and $\varphi$, are more fundamental than $\vec{E}$ and $\vec{B}$. For this reason, we describe the electromagnetic interaction in terms of these potentials, rather than the electric and magnetic fields. This is apparent, at the quantum level, in the Aharonov-Bohm effect where when two beams of electrons are sent through region with same $\vec{B}$, but different $\vec{A}$, experience an interference pattern when collected at the detector (Aharonov \& Bohm, 1959). This occurs because the phase factor of the electron wavefunction depends on $\vec{A}$.

Substituting eq.(3) in (1) and (2), we obtain (Griffiths, 1987)

$$
\frac{1}{c^{2}} \frac{\partial^{2} \varphi}{\partial t^{2}}-\nabla^{2} \varphi-\frac{\partial}{\partial t}\left(\vec{\nabla} \cdot \vec{A}+\frac{1}{c^{2}} \frac{\partial \varphi}{\partial t}\right)=\frac{\rho}{\varepsilon_{0}}
$$

and

$$
\frac{1}{c^{2}} \frac{\partial^{2} \vec{A}}{\partial t^{2}}-\nabla^{2} \vec{A}+\vec{\nabla}\left(\vec{\nabla} \cdot \vec{A}+\frac{1}{c^{2}} \frac{\partial \varphi}{\partial t}\right)=\mu_{0} \vec{J}
$$

These are the Maxwell's equations in terms of the vector and scalar potentials.

\subsection{Lorenz Gauge}

The electric and magnetic fields are invariant under the gauge transformations,

$$
\vec{A}^{\prime}=\vec{A}+\vec{\nabla} \Lambda, \quad \varphi^{\prime}=\varphi-\frac{\partial \Lambda}{\partial t} .
$$

This allows the freedom of taking the (Lorenz gauge) condition,

In this case one has the condition that

$$
\vec{\nabla} \cdot \vec{A}+\frac{1}{c^{2}} \frac{\partial \varphi}{\partial t}=0 .
$$

$$
\frac{1}{c^{2}} \frac{\partial \Lambda}{\partial t}-\nabla^{2} \Lambda=0
$$

It is interesting to see that the electric and magnetic fields can not be uniquely specified by $\vec{A}$ and $\varphi$. For this reason these two fields are treated as mere mathematical constructs and bear no physical meaning, but $\vec{E}$ and $\vec{B}$ do.

\section{Vlaenderen and Waser Approach}

Vlaenderen and Waser have recently adopted a biquaternionic formulation of Maxwell's equation and introduced a scalar function $(S)$ that is a measure of the violation of Lorenz gauge (van Vlaenderen \& Waser, 2001). They remarked that this scalar field mimics the Maxwell's displacement current. For this case, they assumed that

$$
\vec{\nabla} \cdot \vec{A}+\frac{1}{c^{2}} \frac{\partial \varphi}{\partial t}=S
$$

Accordingly, they obtain the following equations

$$
\vec{\nabla} \cdot \vec{E}=\frac{\rho}{\varepsilon_{0}}-\frac{\partial S}{\partial t}, \quad \vec{\nabla} \cdot \vec{B}=0 .
$$

and

$$
\vec{\nabla} \times \vec{E}=-\frac{\partial \vec{B}}{\partial t}, \quad \vec{\nabla} \times \vec{B}=\mu_{0} \vec{J}+\frac{1}{c^{2}} \frac{\partial \vec{E}}{\partial t}+\vec{\nabla} S .
$$

The scalar $S$ satisfies the wave equation

$$
\frac{1}{c^{2}} \frac{\partial^{2} S}{\partial t^{2}}-\nabla^{2} S=\mu_{0}\left(\frac{\partial \rho}{\partial t}+\vec{\nabla} \cdot \vec{J}\right)=0,
$$

upon using the continuity equation. Besides this, $S$ is found to represent an electroscalar wave having a Poynting vector $S \vec{E}$, i.e., along the electric field direction.

\section{Proca's Equations}

Proca generalized Maxwell's equations by introducing a massive photon filed. The resulting equations are (Proca, 1936)

$$
\vec{\nabla} \cdot \vec{E}=\frac{\rho}{\varepsilon_{0}}-\mu_{\gamma}^{2} \varphi, \quad \vec{\nabla} \cdot \vec{B}=0,
$$

and

$$
\vec{\nabla} \times \vec{E}=-\frac{\partial \vec{B}}{\partial t}, \quad \vec{\nabla} \times \vec{B}=\mu_{0} \vec{J}+\frac{1}{c^{2}} \frac{\partial \vec{E}}{\partial t}-\mu_{\gamma}^{2} \vec{A}
$$




\section{Universal Quantum Equations}

We have recently introduced a system of unified quantum wave equations describing matter waves. Like Maxwell's formulation, in this formulation the particle is described by scalar and vector potentials. These equations read (Arbab, 2010)

$$
\frac{1}{c^{2}} \frac{\partial^{2} \psi}{\partial t^{2}}-\nabla^{2} \psi+\frac{2 m}{\hbar} \frac{\partial \psi}{\partial t}+\frac{m^{2} c^{2}}{\hbar^{2}} \psi=0,
$$

and

$$
\frac{1}{c^{2}} \frac{\partial^{2} \vec{\psi}}{\partial t^{2}}-\nabla^{2} \vec{\psi}+\frac{2 m}{\hbar} \frac{\partial \vec{\psi}}{\partial t}+\frac{m^{2} c^{2}}{\hbar^{2}} \vec{\psi}=0 .
$$

Here $\psi$ and $\vec{\psi}$ are the scalar and vector fields (potentials) representing the material wave nature endorsed by de Broglie of a given particle (mass). We assume that any wave that carries a material nature should satisfy these equations. When $m=0$ the material nature disappears and we obtain a wave traveling at the speed of light.

The Lorentz gauge keeps equations Lorentz invariant under Lorentz transformation. Equations $(15,16)$ are Lorentz breaking equations because of the first order time derivative implying that the Lorentz footing of coordinates is broken. However, these equations are invariant under other class of transformation as will be demonstrated in the next section.

\section{The Analogy Among these Paradigms}

Comparing eqs.(10) \& (11) with eqs.(13) \& (14) yields (Note 1)

$$
\mu_{\gamma}^{2} \varphi=-\frac{\partial S}{\partial t}, \quad \quad \mu_{\gamma}^{2} \vec{A}=\vec{\nabla} S .
$$

Differentiation partially the first equation w.r.t. $t$, taking the divergence of the second equation and subtracting the resulting two equations yield

$$
\frac{1}{c^{2}} \frac{\partial^{2} S}{\partial t^{2}}-\nabla^{2} S+\mu_{\gamma}^{2} S=0 .
$$

This is the familiar Klein-Gordon equation of massive spin zero particle. Thus, if the photon had a non-zero mass, as hypothesized by Proca, then this photon wave must be governed by eq.(18).

Integrating the second equation in eq.(17) yields

$$
S=\int \mu_{\gamma}^{2} \vec{A} \cdot \overrightarrow{d r} .
$$

But from Stokes theorem, $\int \vec{A} \cdot \overrightarrow{d r}=\int \vec{\nabla} \times \vec{A} \cdot d \vec{S}=\phi_{m}$, where $\phi_{m}$ is the magnetic flux, one has

$$
S=\mu_{\gamma}^{2} \phi_{m}+f(t) \text {. }
$$

Therefore, eq.(17) can be written as

$$
\varphi=-\frac{\partial \phi_{m}}{\partial t}-\frac{\partial f}{\partial t}
$$

we consider here $f(t)=$ constant. The above equation is nothing but the electromotive force. Hence, the scalar potential $\varphi$ represents the electromotive force. It is evident from eq.(20) that if the Lorenz gauge is satisfied, then the photon must be massless $\left(\mu_{\gamma}^{2}=0\right)$, or otherwise $\phi_{m}=0$. Moreover, the non-satisfaction of Lorenz gauge would lead to massive photon and non-zero magnetic flux. Equation (18) and (20) imply that the magnetic flux satisfies the Klein-Gordon equation, where the flux has a mass, $\mu_{\gamma}^{2}$. Thus, the magnetic flux would propagate as a bosonic particle with spin zero. If we assume now the scalar and vector potentials satisfy the matter wave equation, i.e., eq.(15), then

$$
\vec{\nabla} \cdot \vec{A}+\frac{1}{c^{2}} \frac{\partial \varphi}{\partial t}=-\frac{2 m}{\hbar} \varphi .
$$

This equation can be compared with eq.(9), in which case, $S=-\frac{2 m}{\hbar} \varphi$. If we compare eqs.(9), (17), (18) and (22) we will get

$$
\frac{1}{c^{2}} \frac{\partial^{2} \varphi}{\partial t^{2}}-\nabla^{2} \varphi+\mu_{\gamma}^{2} \varphi=0 .
$$

This implies that the scalar potential of the massive photon, $\varphi$, satisfies the Klein-Gordon equation.

Now differentiating partially eq.(22) with respect to time and using (3) yield

$$
\frac{1}{c^{2}} \frac{\partial^{2} \varphi}{\partial t^{2}}-\nabla^{2} \varphi+\frac{2 m}{\hbar} \frac{\partial \varphi}{\partial t}-\vec{\nabla} \cdot \vec{E}=0
$$


Taking the gradient of eq.(22) and using eq.(3) and the second equation in eq.(2), we obtain

$$
\frac{1}{c^{2}} \frac{\partial^{2} \vec{A}}{\partial t^{2}}-\nabla^{2} \vec{A}+\frac{2 m}{\hbar} \frac{\partial \vec{A}}{\partial t}+\frac{2 m}{\hbar} \vec{E}-\mu_{0} \vec{J}=0,
$$

where we have used the vector identity, $\vec{\nabla} \times(\vec{\nabla} \times \vec{A})=\vec{\nabla}(\vec{\nabla} \cdot \vec{A})-\nabla^{2} \vec{A}$.

It is interesting to see that if the photon had a mass $(m \neq 0)$ in vacuum, then the Lorenz gauge in eq.(22) would not be satisfied. This encourages us to associate a non-zero mass of the photon to the breaking of Lorenz gauge.

In vacuum, $\rho=0$ and $\vec{J}=0$, so that eqs.(24) and (25), using eq.(3), become

$$
\frac{1}{c^{2}} \frac{\partial^{2} \varphi}{\partial t^{2}}-\nabla^{2} \varphi+\frac{2 m}{\hbar} \frac{\partial \varphi}{\partial t}=0 .
$$

Taking the gradient of eq.(22) and using eq.(3) and the second equation in eq.(2), we obtain

$$
\frac{1}{c^{2}} \frac{\partial^{2} \vec{A}}{\partial t^{2}}-\nabla^{2} \vec{A}-\frac{2 m}{\hbar} \vec{\nabla} \varphi=0 .
$$

Therefore, eqs.(26) and (27) are the corresponding equations describing massive photon in vacuum. Adding the time derivative of eq.(27) to the gradient of eq.(26), using eq.(3), imply that both $\vec{E}$ and $\vec{B}$ travel at speed of light, viz.,

$$
\frac{1}{c^{2}} \frac{\partial^{2} \vec{E}}{\partial t^{2}}-\nabla^{2} \vec{E}=0, \quad \frac{1}{c^{2}} \frac{\partial^{2} \vec{B}}{\partial t^{2}}-\nabla^{2} \vec{B}=0 .
$$

This is a quite surprising result. It is evident from eqs.(26) and (27) that spatial and temporal variations of the scalar field is the source of the $\varphi$ and $\vec{A}$ waves.

Now apply the gauge transformation, eq.(6), in eq.(22) to get

$$
\frac{1}{c^{2}} \frac{\partial^{2} \Lambda}{\partial t^{2}}-\nabla^{2} \Lambda+\frac{2 m}{\hbar} \frac{\partial \Lambda}{\partial t}=0 .
$$

This equation is know as the Telegrapher's equation. It describes the electric signals in telephone and telegraph lines. However, by making the substitution, $\Lambda(r, t)=e^{-\left(m c^{2} t / \hbar\right)} \chi(r, t)$, eq. (29) can be written as

$$
\frac{1}{c^{2}} \frac{\partial^{2} \chi}{\partial t^{2}}-\nabla^{2} \chi+\frac{m^{2} c^{2}}{\hbar^{2}} \chi=0 .
$$

which is the ordinary Klein-Gordon equation of massive scalar field $\chi$. This equation is a consequence of gauge invariance.

\subsection{The Combined Gauge Transformation}

We will consider here the combined gauge transformations in which both $\varphi \& \vec{A}$, as well as, $\rho \& \vec{J}$ are transformed. Interestingly, Proca equations are invariant under these combined gauge transformations. These are given by

$$
\vec{A}^{\prime}=\vec{A}+\vec{\nabla} \Lambda, \quad \varphi^{\prime}=\varphi-\frac{\partial \Lambda}{\partial t},
$$

and

$$
\vec{J}^{\prime}=\vec{J}+\mu_{\gamma}^{2} \varepsilon_{0} c^{2} \vec{\nabla} \Lambda, \quad \quad \rho^{\prime}=\rho-\mu_{\gamma}^{2} \varepsilon_{0} \frac{\partial \Lambda}{\partial t} .
$$

Under these gauge transformations, Proca's equations become gauge invariant and that the mass term is no longer problematic. Moreover, these transformations leave the system of the continuity equations invariant as well, as long as $\Lambda$ satisfies the wave equation in eq.(8) (Arbab \& Widatallah, 2010).

\section{The Status of Experimental Evidence of Longitudinal Wave and Photon Mass}

There are some appealing experiments that amount to the existence of longitudinal wave. An electric longitudinal wave has been observed by Miyaji et al. while studying the transverse wave (Miyaji et al., 2004). This wave is characterized by the vanishing magnetic component.

The enormous success in quantum electrodynamics has lead to the acceptance of the concept of massless photons. Also, the nonzero photon mass would give rise to a wavelength dependence of the speed of light in free space, a more rapid (exponential or Yukawa) falloff of magnetic dipole fields with distance than the usual inverse cube dependence (Barrow 
\& Burman, 1984). This could be attributed to the possibility of existing longitudinal electromagnetic waves. This wave is characterized by the vanishing magnetic and electric field components (Comay, 1996). A massive photon is equivalent to a violation of Coulomb's Law (Tu \& Luo, 2004). Hence, a Yukawa factor $e^{-r / \lambda}$ in the $1 / r$ electrostatic and magnetostatic potential terms would appear. The magnetic field term will contain correction terms related to the de Broglie wavelength $\lambda_{\gamma}=\hbar / m_{\gamma} c$. Another way to test the existence of the photon mass and hence the longitudinal waves is the limit of the power in the inverse of the distance distance in Coulomb law, namely $1 / r^{2+\varepsilon}$. One of the consequences of $\varepsilon \neq 0$ is the decrease of the electromagnetic waves as the wavelength increases which is due to the smallness of $\varepsilon$. A limit of $\varepsilon=5.1 \times 10^{-20}$ was set by Lou et al (Spavieri, Gillies, \& Rodriguez, 2004). According to the uncertainty principle the upper limit on the photon mass can be obtained from $m_{\gamma} \approx \hbar /\left(\Delta t c^{2}\right)$. Using the age of the universe as $10^{10}$ years one obtains $m_{\gamma} \approx 10^{-66} \mathrm{~g}$.

De Broglie noticed that photon mass leads to a larger speed of violet light than that of the red one (de Broglie, 1942). He concluded that during the eclipse in a double star system the color of the appearing star would change from violet to red. On the other hand, Schrodinger pointed out that magnetic field of the Earth would be exponentially cut off at distances of the order of the photon Compton wave length (Schr?dinger, 1943). From the observed altitude of auroras he concluded that $\lambda_{y}>10^{4}$. Other data have shown more limits also.

Because of limitations involved in experiments performed on the Earth, astrophysical measurement could promise better limits on photon masses. One of the consequences of photons being massive is the dispersion of star light. According to measurements performed on planet Jupiter the upper limit of Compton wavelength is $\lambda_{\gamma}=3.14 \times 10^{11} \mathrm{~cm}(\mathrm{Gintsburg}$, 1964). Based on analyzing the Earth's magnetic field the upper limit on photon mass is $m_{\gamma}=1 \times 10^{-48} \mathrm{~g}(\mathrm{Chibisov}, 1976)$. Also from geomagnetic field Fischbach et al. found a limit on the upper mass of the photon as $m_{\gamma} \leq 10^{-48} \mathrm{~g}$ (Fischbach et al., 1986). According to Proca equation, the velocity of light depends on its frequency. From the measurement on the radio signals from the Crab Nebula it was found that the upper limit on the mass of the photon is $m_{\gamma} \leq 10^{-44} \mathrm{~g}$ (Tu, Luo, \& Gillies, 2004). Also, hydrodynamic waves coupling to the interplanetary magnetic fields could be useful in finding the mass of the photon. Ryutov (1997) set a limit of $m_{\gamma} \leq 10^{-51} \mathrm{~g}$ based on these studies (Ryutov, 1997). Other limits on the photon mass are obtained from studying the current density and plasma of the interstellar media $\left(m_{\gamma} \leq 10^{-53} \mathrm{~g}\right)(\mathrm{Ryutov}$, 2007).

Modern theories that appeal to the concept of spontaneous symmetry breaking assume that particles, which are massless above a certain critical temperature $T_{c}$, acquire mass below this temperature. This can be applied to photons. From the cryogenic photon mass experiment performed by Ryan et al an upper limit mass on photons were found to be $m_{\gamma}=$ $1.5 \times 10^{-42}$. From the symmetry breaking point of view and the experiment, one can speculate that photons can be massless above a certain temperature (Ryan, Accetta, \& Austin, 1985).

Superconductivity is characterized by perfect conductivity and perfect diamagnetism. BCS theory can explain both of these fundamental properties of superconductors (Bardeen, Cooper, \& Schrieffer, 1957). However, the London equations can be easily derived and used to understand the expulsion of flux within the material without appealing to the broader BCS theory (London \& London, 1935). Such a derivation relies solely on postulating that the Cooper pairs in the superconducting state maintain their ground state momentum of $p=0$. It has been possible to identify an effective photon mass that is directly related to the London penetration depth of the applied field (Tajmar \& De Matos, 2006).

In 2008 a Russian group performed a measurement on the volt value triggered by the radiation coming from solar eclipse. It was noticed the registered signal does not depend on the orientation of the tourmaline plates used in front of the detector (Zaymidoroga \& Podgainy, 2011). This could be an indication of the longitudinal wave component and, hence, the non-zero mass of the photon.

Another experimental evidence for the existence of the massive photons is the Tesla coil. The coil is a device which produces high frequency alternating current with high voltage and low current. The coil is used to produce energy which can be transmitted to a device without any wires. A neon light can lit without wiring connection. This can be explained in terms of the longitudinal component of the light wave.

\section{Conclusion}

In this paper, the possibility of longitudinal photon wave is explored from the electromagnetic theory. We revisited Proca, Vlaenderen \& Waser and Arbab theories. We studied the equations obtained from these approaches and combined them together. The different approaches are compared to each other and the relevant equations are combined. It is shown, then, that if the Lorentz gauge invariance is violated the photon can gain mass and the longitudinal part of the electromagnetic wave can appear. 


\section{References}

Aharonov, Y., \& Bohm, D. (1959). Significance of electromagnetic potentials in the quantum theory. Physical Review, $115(3), 485$.

Arbab, A. I. (2010). Derivation of Dirac, Klein-Gordon, Schr?dinger, diffusion and quantum heat transport equations from a universal quantum wave equation. EPL (Europhysics Letters), 92(4), 40001.

Arbab, A. I., \& Satti, Z. A. (2009). On the generalized Maxwell equations and their prediction of electroscalar wave. Progress in physics, 2(8), 8-13.

Arbab, A. I., \& Widatallah, H. M. (2010). On the generalized continuity equation. Chinese Physics Letters, 27(8), 084703.

Bardeen, J., Cooper, L. N., \& Schrieffer, J. R. (1957). Theory of superconductivity. Physical Review, 108(5), 1175.

Barrow, J. D., \& Burman, R. R. (1984). Particle physics and cosmology: New light on heavy light. Nature, 307(5946), 14.

Bass, L., \& Schr?dinger, E. (1955). Must the photon mass be zero?. Proc. R. Soc. Lond. A, 232(1188), 1-6.

Chibisov, G. V. (1976). Astrophysical upper limits on the photon rest mass. Soviet Physics Uspekhi, $19(7), 624$.

Comay, E. (1996). Comment on the longitudinal magnetic field of circularly polarized electromagnetic waves. Chemical physics letters, 261(4-5), 601-604.

de Broglie, L. (1942). La mcanique ondulatoire du photon: Les interactions entre les photons et la matire (Vol. 2). Hermann.

Fischbach, E., Sudarsky, D., Szafer, A., Talmadge, C., \& Aronson, S. H. (1986). Reanalysis of the Eoumltv?s experiment. Physical Review Letters, 56(1), 3 .

Gintsburg, M. A. (1964). Astronom. Zhurnal 40, 703 (1963) MA Gintsburg. Sov. Astron, 7, 536. (in Russian)

Glashow, S. L. (1961). Partial-symmetries of weak interactions. Nuclear Physics, 22(4), 579-588.

Griffiths, D. (1987). Introduction to Electrodynamics. John Wiley \& Sons.

London, F., \& London, H. (1935). The electromagnetic equations of the supraconductor. Proc. R. Soc. Lond. A, 149(866), 71-88.

Miyaji, G., Miyanaga, N., Tsubakimoto, K., Sueda, K., \& Ohbayashi, K. (2004). Intense longitudinal electric fields generated from transverse electromagnetic waves. Applied physics letters, 84(19), 3855-3857.

Proca, A. L. (1936). Sur la thorie ondulatoire des electrons positifs et negatifs. Journal de Physique et le Radium, 7(8), 347-353.

Ryan, J. J., Accetta, F., \& Austin, R. H. (1985). Cryogenic photon-mass experiment. Physical Review D, 32 (3), 802.

Ryutov, D. D. (1997). The role of finite photon mass in magnetohydrodynamics of space plasmas. Plasma Physics and Controlled Fusion, 39(5A), A73.

Ryutov, D. D. (2007). Using plasma physics to weigh the photon. Plasma Physics and Controlled Fusion, $49(12 B)$, B429.

Salam, A. (1962). Renormalizability of gauge theories. Physical Review, 127(1), 331.

Schr?dinger, E. (1943, January). The earth's and the sun's permanent magnetic fields in the unitary field theory. In Proceedings of the Royal Irish Academy. Section A: Mathematical and Physical Sciences (Vol. 49, pp. 135-148). Royal Irish Academy.

Spavieri, G., Gillies, G. T., \& Rodriguez, M. (2004). Physical implications of Coulomb's Law. Metrologia, $41(5)$, S159.

Tajmar, M., \& De Matos, C. J. (2006). Local photon and graviton mass and its consequences. arXiv preprint grqc/0603032.

Tu, L. C., \& Luo, J. (2004). Experimental tests of Coulomb's Law and the photon rest mass. Metrologia, $41(5)$, S136.

Tu, L. C., Luo, J., \& Gillies, G. T. (2004). The mass of the photon. Reports on Progress in Physics, $68(1), 77$.

van Vlaenderen, K. J., \& Waser, A. (2001). Generalisation of classical electrodynamics to admit a scalar field and longitudinal waves. Hadronic Journal, 24(5), 609-628.

Weinberg, S. (1967). WEINBERG 1967. Phys. Rev. Lett, 19, 1264. 
Zaymidoroga, O. A., \& Podgainy, D. V. (2011). Observation of electroscalar radiation during a solar eclipse. In Cosmic Rays For Particle And Astroparticle Physics (pp. 84-89).

Note

Note 1 . We consider here $-S$ instead of $S$

\section{Copyrights}

Copyright for this article is retained by the author(s), with first publication rights granted to the journal.

This is an open-access article distributed under the terms and conditions of the Creative Commons Attribution license (http://creativecommons.org/licenses/by/4.0/). 